\title{
Newtonian pizza: spinning a viscous sheet
}

\author{
PETER D. HOWELL ${ }^{1}$, BENOIT SCHEID ${ }^{2,3} \dagger$ \\ AND HOW ARD A. STONE S $^{2,4}$ \\ ${ }^{1}$ Mathematical Institute, University of Oxford, 24-29 St Giles, Oxford OX1 3LB, UK \\ ${ }^{2}$ School of Engineering and Applied Sciences, Harvard University, Cambridge, MA 02138, USA \\ ${ }^{3}$ TIPs - Fluid Physics unit, Université Libre de Bruxelles, C.P. 165/67, 1050 Brussels, Belgium \\ ${ }^{4}$ Department of Mechanical and Aerospace Engineering, Princeton University, Princeton, NJ 08544, USA
}

(Received 20 April 2009; revised 27 March 2010; accepted 29 March 2010; first published online 2 August 2010)

We study the axisymmetric stretching of a thin sheet of viscous fluid driven by a centrifugal body force. Time-dependent simulations show that the sheet radius $R(t)$ tends to infinity in finite time. As time $t$ approaches the critical time $t^{*}$, the sheet becomes partitioned into a very thin central region and a relatively thick rim. A net momentum and mass balance in the rim leads to a prediction for the sheet radius near the singularity that agrees with the numerical simulations. By asymptotically matching the dynamics of the sheet with the rim, we find that the thickness $h$ in the central region is described by a similarity solution of the second kind, with $h \propto\left(t^{*}-t\right)^{\alpha}$ where the exponent $\alpha$ satisfies a nonlinear eigenvalue problem. Finally, for non-zero surface tension, we find that the exponent increases rapidly to infinity at a critical value of the rotational Bond number $B=1 / 4$. For $B>1 / 4$, surface tension defeats the centrifugal force, causing the sheet to retract rather than to stretch, with the limiting behaviour described by a similarity solution of the first kind.

Key words: lubrication theory, thin films

\section{Introduction}

Numerous processes in industry and nature depend on stretching thin sheets of viscous liquid. Specific industrial applications include polymer film casting and the float glass process (see e.g. Pearson 1985 and the references therein). On a much larger scale, the Earth's lithosphere behaves as a thin viscous sheet over geological time scales (England \& McKenzie 1982). Though the stretching of liquid sheets in many industrial applications is essentially unidirectional, two-dimensional stretching is also used in practice (Pilkington 1969), as recently modelled by Scheid et al. (2009). In either case, the main driving force is set by the boundary conditions, though gravity if aligned with the stretching direction can also contribute to the thinning process (Cao, Khayat \& Puskas 2005). In this paper, we investigate the use of centrifugal force to stretch a thin circular viscous sheet, inspired by the example of a chef spinning pizza dough around his hand. For simplicity, we limit our study to incompressible Newtonian fluid, although pizza dough is in reality both viscoelastic and shear-thinning (Ng, McKinley \& Padmanabhan 2006). 
The radial expansion of circular sheets has been widely studied in the context of atomization since the pioneering work by Savart (1833) and later Taylor $(1959 a, b)$ and Huang (1970). An axisymmetric liquid sheet may be formed by letting a round liquid jet impact a flat circular surface. As the sheet expands, the fluid collects in a rim around the edge, which eventually breaks up into small droplets (e.g. Eggers \& Villermaux 2008). The surface-tension-driven radial retraction of fluid sheets is also an active research topic in the context of spray formation as well as in the rupture of a soap film or a liquid curtain (e.g. Savva \& Bush 2009). The theoretical framework for modelling thin viscous sheets can been found, for instance, in Buckmaster, Nachman \& Ting (1975), Howell (1996) or Ribe (2002).

The topic of finite-time rupture has been treated by many authors, such as Erneux \& Davis (1993), Ida \& Miksis (1996) and Vaynblat, Lister \& Witelski (2001a) for viscous sheets and Papageorgiou (1995), Eggers (1997) and Renardy (2001) for viscous threads. Vaynblat, Lister \& Witelski (2001b) have shown that lubrication models for van der Waals driven rupture of a sheet and capillary driven rupture of a thread both give rise to families of first-kind similarity solutions. Similarity solution of the second kind - i.e. with non-rational scaling exponents as classified by Barenblatt (1996) was suggested in a study of sheet rupture by Ida \& Miksis (1996) in a transient regime where van der Waals and viscous forces dominate the evolution. However, Vaynblat et al. (2001a) demonstrated that the eventual asymptotic dynamical balance is between van der Waals forces, viscosity and inertia, which then gives rise to a symmetric first-kind similarity solution. Vaynblat et al. (2001b) reached the same conclusion for the breakup of a viscous thread, though in this case the first-kind similarity solution is asymmetric. By neglecting inertia, Papageorgiou (1995) found similarity solutions of the second kind valid as the radius of a viscous thread tends to zero after a finite time. Similarly, we will show that a spinning viscous sheet stretches to infinity in finite time, with the ultimate behaviour described by a similarity solution of the second kind. We will find that the centrifugal force sends the sheet thickness to zero everywhere simultaneously, in contrast with van der Waals interaction (in the case of a sheet) and capillary stress (in the case of a thread), both of which cause finite-time rupture at a single point.

In $\S 2$ we pose the mathematical formulation and perform time-dependent simulations of a spinning viscous sheet. In $\S 3$ we undertake small-time asymptotics to understand the early stages of the expansion. In $\S 4$ we show that a similarity solution of the first kind cannot satisfy all the boundary conditions and therefore fails to describe the limiting behaviour. In $\S 5$ we describe an asymptotic decomposition into a flat central region and a thicker rim, which occurs at later times of the expansion. This approach motivates us to perform in $\S 6$ an asymptotic analysis of each region separately and thus determine the matching conditions. We show that the central region is described by a similarity solution of the second kind, in which the exponent is found by satisfying the matching conditions with the rim. In $\S 7$ we generalize our results to include surface tension. Our conclusions are presented in $\S 8$.

\section{Problem formulation}

\subsection{Stress balance}

We consider a circular viscous sheet of time-dependent radius $R(t)$ spinning at angular rate $\Omega$ and with constant density $\rho$ and viscosity $\mu$. A cylindrical $(r, \theta, z)$ coordinate system is chosen with the origin at the centre of the sheet, as sketched in figure 1 . The flow is assumed extensional and axisymmetric, and is therefore fully described 


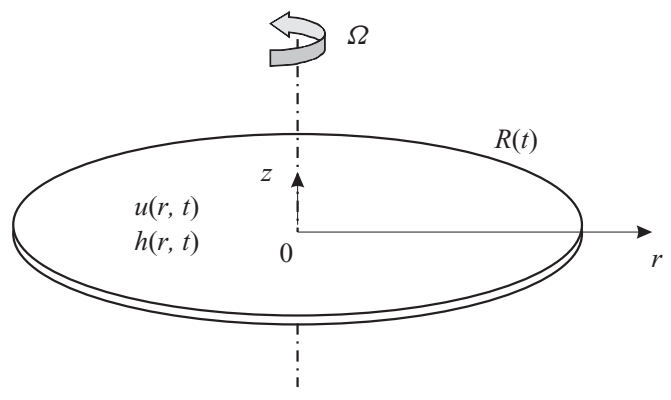

FIGURE 1. Sketch of a spinning viscous sheet.

by the radial velocity $u=u(r, t)$ and the sheet thickness $h=h(r, t)$. This description remains valid as long as $h \ll R$ and prior to any azimuthal instability.

For a radial extensional flow, the radial and azimuthal stresses are given respectively by

$$
\sigma_{r r}=-p+2 \mu \frac{\partial u}{\partial r} \quad \text { and } \quad \sigma_{\theta \theta}=-p+2 \mu \frac{u}{r} .
$$

The pressure $p$ is obtained from the vanishing of the transverse stress $\sigma_{z z}$, which leads to

$$
p=-\frac{2 \mu}{r} \frac{\partial(r u)}{\partial r} .
$$

If we ignore inertia, surface tension and intermolecular forces, then a balance between extensional and centrifugal stresses averaged across the thickness of the sheet gives

$$
\frac{1}{r} \frac{\partial\left(r h \sigma_{r r}\right)}{\partial r}-\frac{h \sigma_{\theta \theta}}{r}=-\rho \Omega^{2} r h .
$$

Here we have neglected all fluid inertia, including Coriolis effects, but retained the centrifugal acceleration. This assumption is valid provided the rotational Reynolds number $\rho \Omega R^{2} / \mu$ is small. Although the use of a Newtonian constitutive relation is questionable for pizza dough, we can use the results of $\mathrm{Ng}$ et al. (2006) to estimate a typical value of the viscosity $\mu \approx 10^{4} \mathrm{~Pa}$. Hence, with $\rho \approx 10^{3} \mathrm{~kg} \mathrm{~m}^{-3}, \Omega \approx 2 \pi \mathrm{s}^{-1}$ and $R \approx 0.15 \mathrm{~m}$ for a 12 in. pizza, we find that $\rho \Omega R^{2} / \mu \approx 0.014$.

\subsection{Dimensionless equations}

Defining the characteristic timescale $\bar{\tau}=\mu / \rho \Omega^{2} \bar{R}^{2}$ over which the stress balance (2.3) takes place, and using the initial radius $\bar{R}$ and the average initial thickness $\bar{h}$, the non-dimensionalization is performed through the transformations

$$
r \rightarrow \bar{R} r, \quad R \rightarrow \bar{R} R, \quad t \rightarrow \bar{\tau} t, \quad h \rightarrow \bar{h} h, \quad u \rightarrow \frac{\bar{R}}{\bar{\tau}} u \quad \text { and } \quad \sigma \rightarrow \frac{\mu}{\bar{\tau}} \sigma .
$$

The dimensionless stress balance (2.3) then becomes

$$
\frac{\partial}{\partial r}\left(r h \sigma_{r r}\right)-h \sigma_{\theta \theta}=-h r^{2}
$$

and use of (2.2) reduces the dimensionless constitutive relations (2.1) to

$$
\sigma_{r r}=4 \frac{\partial u}{\partial r}+2 \frac{u}{r} \quad \text { and } \quad \sigma_{\theta \theta}=2 \frac{\partial u}{\partial r}+4 \frac{u}{r} .
$$



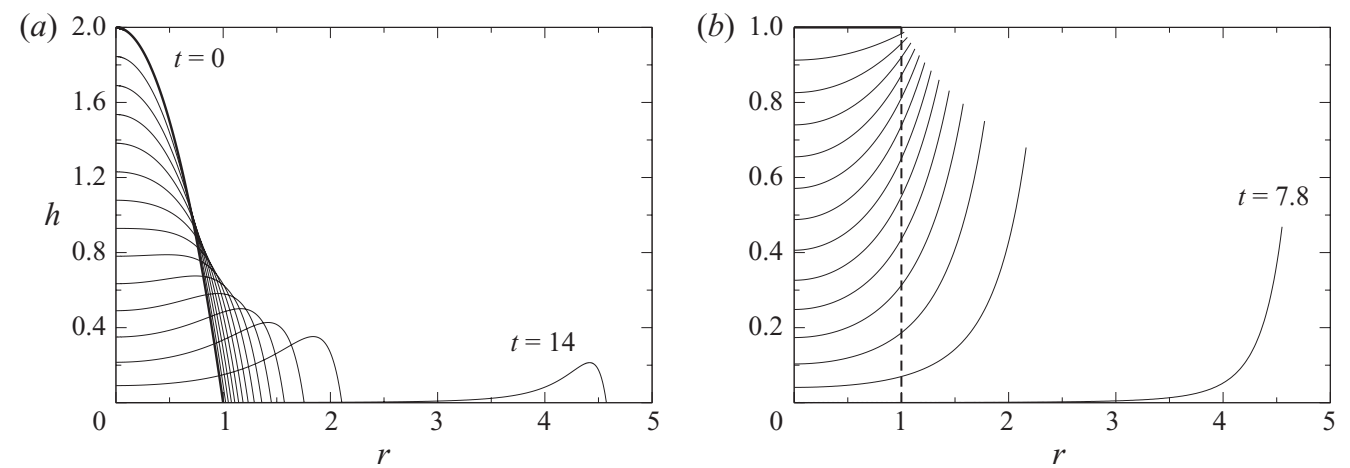

FIGURE 2. Numerical results for the sheet thickness profile $h(r, t)$ versus radial coordinate $r$ for two different initial conditions (thick lines); time interval $\Delta t=1.0$ ('drop'), $\Delta t=0.6$ ('box'). (a) 'Drop' condition : $h_{0}(r)=2\left(1-r^{2}\right) ;(b)$ 'box' condition: $h_{0}(r)=1$.

Substituting (2.6) into (2.5) yields

$$
4 \frac{\partial}{\partial r}\left(\frac{h}{r} \frac{\partial(r u)}{\partial r}\right)-2 \frac{u}{r} \frac{\partial h}{\partial r}=-r h
$$

The system is closed by the mass conservation equation

$$
\frac{\partial}{\partial t}(r h)+\frac{\partial}{\partial r}(r h u)=0
$$

and by symmetry, kinematic and zero-stress boundary conditions:

$$
u(0, t)=0, \quad u(R(t), t)=\dot{R}(t) \quad \text { and } \quad \sigma_{r r}(R(t), t)=0,
$$

where the dot denotes the time derivative. This problem description has no dimensionless parameters and so is completely determined by the initial conditions

$$
h(r, 0)=h_{0}(r) \quad \text { and } \quad R(0)=1 .
$$

The edge $r=R(t)$ will be rounded by a meniscus whose aspect ratio is $O(1)$, so it is not captured by our long-wavelength model. Net mass and stress balances on this meniscus give rise to the effective boundary conditions $(2.9 b, c)$. The stress balance $(2.9 c)$ will be generalized in $\S 7$ to account for surface tension, which introduces a rotational Bond number into the formulation.

\subsection{Numerical simulations}

We first look at time-dependent solutions of the system (2.7)-(2.10), performed by the finite element method using Comsol. The computations are facilitated by performing a Lagrangian transformation, as described in the Appendix, to map the moving domain onto a fixed interval. The mesh size is refined down to $10^{-8}$ in the central region to resolve the singularity in the system of equations as $r \rightarrow 0$.

Because the system is parameter free, the only tunable quantity is the initial thickness profile $h_{0}(r)$. We consider two illustrative examples: a 'drop' initial condition with $h_{0}(r)=2\left(1-r^{2}\right)$ and a 'box' condition with $h_{0}(r)=1$. The numerical results are plotted in figure 2, which shows thickness profiles for regular time intervals from $t=0$ (thick lines) until a time close to the critical time $t=t^{*}$ at which the sheet radius $R(t)$ tends to infinity and the middle thickness $h(0, t)$ tends to zero (within the limits of numerical accuracy). 
These simulations show that for either initial condition two different regimes in the time evolution may be identified. Initially, the film thins at an essentially constant rate, with the radius still close to $R \sim 1$. At later times, the fluid accumulates in a thick rim, leaving a very thin film in the centre, while the radius expands to infinity in finite time. We will show below how these observations can be explained using asymptotic analysis.

\section{Small-time asymptotics}

To investigate the early stages of the sheet evolution, we seek a small- $t$ expansion of the form

$h(r, t) \sim h_{0}(r)+t h_{1}(r)+\cdots, \quad u(r, t) \sim u_{0}(r)+t u_{1}(r)+\cdots, \quad R(t) \sim 1+t R_{1}+\cdots$.

The initial thickness $h_{0}(r)$ is specified, while the momentum equation (2.7) leads to an ordinary differential equation (ODE) for the initial velocity $u_{0}(r)$, namely

$$
u_{0}^{\prime \prime}+\left(\frac{1}{r}+\frac{h_{0}^{\prime}}{h_{0}}\right) u_{0}^{\prime}+\left(\frac{h_{0}^{\prime}}{2 r h_{0}}-\frac{1}{r^{2}}\right) u_{0}=-\frac{r}{4},
$$

subject to the boundary conditions

$$
u_{0}(0)=0 \quad \text { and } \quad 4 u_{0}^{\prime}(1)+2 u_{0}(1)=0,
$$

where a prime denotes the $r$-derivative. The first-order thickness correction $h_{1}(r)$ is then found from (2.8) to be given by

$$
h_{1}=-\frac{1}{r}\left(r h_{0} u_{0}\right)^{\prime}
$$

while the kinematic boundary condition $(2.9 b)$ determines the initial rim velocity as

$$
R_{1}=u_{0}(1)
$$

Analytical solution of even the leading-order equation (3.2) is unlikely to be feasible unless $h_{0}(r)$ takes a reasonably simple form. The most straightforward case is the 'box' initial condition $h_{0}(r) \equiv 1$, for which the solution of (3.2) and (3.3) is

$$
u_{0}(r)=\frac{r\left(7-3 r^{2}\right)}{96} \text {. }
$$

From (3.4) and (3.5), we then easily find

$$
h_{1}(r)=-\frac{\left(7-6 r^{2}\right)}{48} \quad \text { and } \quad R_{1}=\frac{1}{24} .
$$

The procedure may be continued to find the higher-order corrections:

$$
\begin{aligned}
u(r, t) & \sim \frac{r\left(7-3 r^{2}\right)}{96}+\frac{7 r\left(26-9 r^{2}+3 r^{4}\right) t}{18432}+\frac{7 r\left(885-270 r^{2}+64 r^{4}-19 r^{6}\right) t^{2}}{4718592}+\cdots \\
h(r, t) & \sim 1-\frac{\left(7-6 r^{2}\right) t}{48}+\frac{\left(14-378 r^{2}+153 r^{4}\right) t^{2}}{18432}+\cdots \\
R(t) & \sim 1+\frac{t}{24}+\frac{31 t^{2}}{9216}+\cdots
\end{aligned}
$$



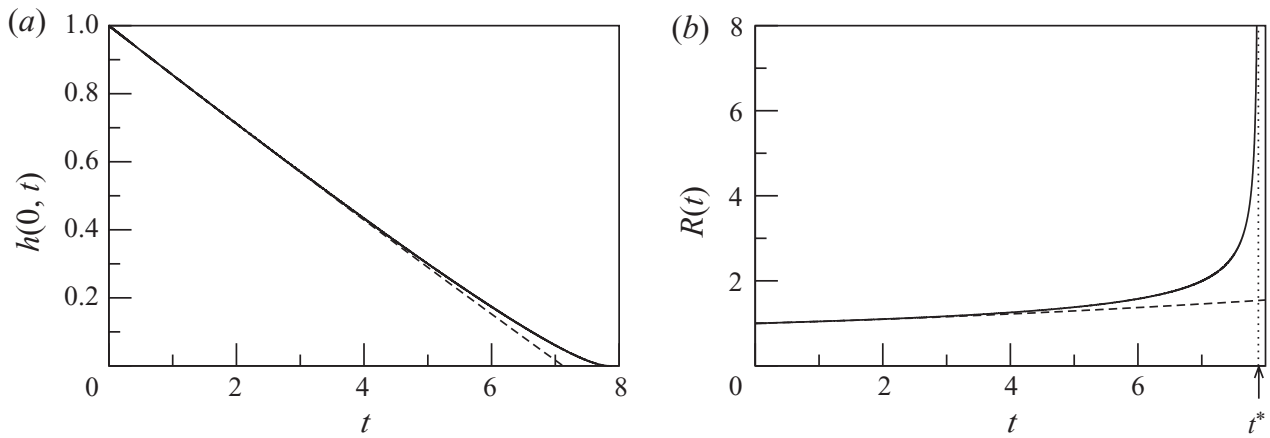

FIGURE 3. Comparison between the simulation (solid line) and the small-time expansion (3.9)-(3.10) (dashed line) for the 'box' initial condition; $t^{*} \approx 7.8878$ indicates the critical time at which $R$ tends to infinity.

These series appear to converge rapidly, and figure 3 confirms that the minimum thickness $h(0, t)$ and sheet radius $R(t)$ are indeed well approximated as linear functions of $t$. However, the approximation obviously fails to describe the singular behaviour of the radius $R(t)$ as $t$ approaches the critical time $t^{*}$, which is investigated in the subsequent sections.

\section{Failure of the first-kind similarity solution}

The simulation results shown in figure 2 indicate that the sheet radius tends to infinity, while the thickness tends to zero, as $t$ approaches some finite time $t^{*}$. We would expect the solution in the neighbourhood of such a singularity to be self-similar. We easily find that the ansatz

$$
h=\left(t^{*}-t\right)^{\alpha} f(\eta), \quad u=\left(t^{*}-t\right)^{-3 / 2} g(\eta), \quad R=\left(t^{*}-t\right)^{-1 / 2} \ell,
$$

where $\eta=r \sqrt{t^{*}-t}$ is the similarity coordinate, balances all the terms in the governing equations (2.7) and (2.8), for any value of the exponent $\alpha$. The net mass-conservation condition

$$
\int_{0}^{R(t)} h(r, t) r \mathrm{~d} r=\left(t^{*}-t\right)^{\alpha-1} \int_{0}^{\ell} f(\eta) \eta \mathrm{d} \eta=\frac{1}{2}
$$

(the value $1 / 2$ following from our non-dimensionalization of $h$ ) requires us to choose $\alpha=1$, and by imposing the condition $g(0)=0$ we quickly obtain the solution

$$
f(\eta)=\frac{\mathrm{e}^{-\eta^{2} / 6}}{6\left(1-\mathrm{e}^{-\ell^{2} / 6}\right)}, \quad g(\eta)=\frac{\eta}{2} .
$$

There are several problems with this similarity solution. First, all of our numerical simulations indicate that the sheet thickness is minimized at the centre as $t \rightarrow t^{*}$, while (4.3) gives a maximum in $h(r, t)$ at $r=0$. In addition, the radial stress corresponding to (4.3), namely $\sigma_{r r}=3 /\left(t^{*}-t\right)$, does not satisfy the zero-stress boundary condition at the edge $r=R(t)$. Finally, we note that our numerical solutions suggest $h(0, t) \propto\left(t^{*}-t\right)^{\alpha}$ with $\alpha \approx 4$ (see $\left.\S 6.5\right)$, not $\alpha=1$.

The similarity solution (4.3) is of the first kind, following the classification by Barenblatt (1996), with rational scaling exponents chosen to balance all the terms in the equations and to conserve mass globally. Thus, we have found that no such solution exists that satisfies the stress-free condition at $r=R(t)$, and a more careful 


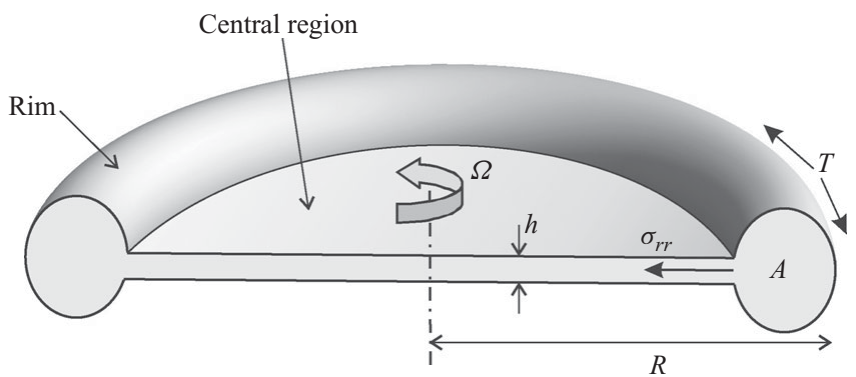

Figure 4. Asymptotic decomposition effective as $t$ approaches the critical time $t^{*} ; A(t)$ is the cross-sectional area of the rim.

analysis is therefore required to determine the correct scaling behaviour in the limit $t \rightarrow t^{*}$.

\section{Asymptotic decomposition}

Our simulations show that, as the critical time $t^{*}$ is approached, the fluid sheet deforms into two distinct domains, as shown schematically in figure 4 . We denote the very thin flat sheet in the centre as the 'central region' and the relatively thick lobe near the edge as the 'rim'. For the sake of clarity, the equations and variables in this section are given in dimensional terms, unless specified otherwise.

As the rim expands (with radius $R(t)$ ), it experiences an extensional strain rate of $\dot{R} / R$. This gives rise to an azimuthal or hoop tension

$$
T=\frac{3 \mu A \dot{R}}{R},
$$

where $A(t)$ is the cross-sectional area, and the factor 3 is the Trouton ratio for the drawing of a viscous thread (Pearson 1985). Meanwhile, the radial stress in the central region is given by

$$
\sigma_{r r}=-\frac{3 \mu \dot{h}}{h},
$$

where we approximate the sheet thickness $h$ as a function only of $t$. A net balance between the centrifugal force, radial stress and hoop tension acting on the rim leads to

$$
\frac{T}{R}+\sigma_{r r} h=\rho \Omega^{2} R A
$$

Net mass conservation gives us one more equation, namely

$$
2 \pi R A+\pi R^{2} h=V,
$$

where $V=\pi \bar{R}^{2} \bar{h}$ is the constant total volume of the sheet.

In (5.1)-(5.4) we have just four equations for the five unknowns $T, \sigma_{r r}, A, R$ and $h$, so more information is needed. We can make progress by supposing that the stress $\sigma_{r r}$ exerted by the central region on the rim is negligible; this assumption will be justified a posteriori. In this limit, (5.1) and (5.3) reduce to the differential equation

$$
\frac{\dot{R}}{R^{3}}=\frac{\rho \Omega^{2}}{3 \mu},
$$



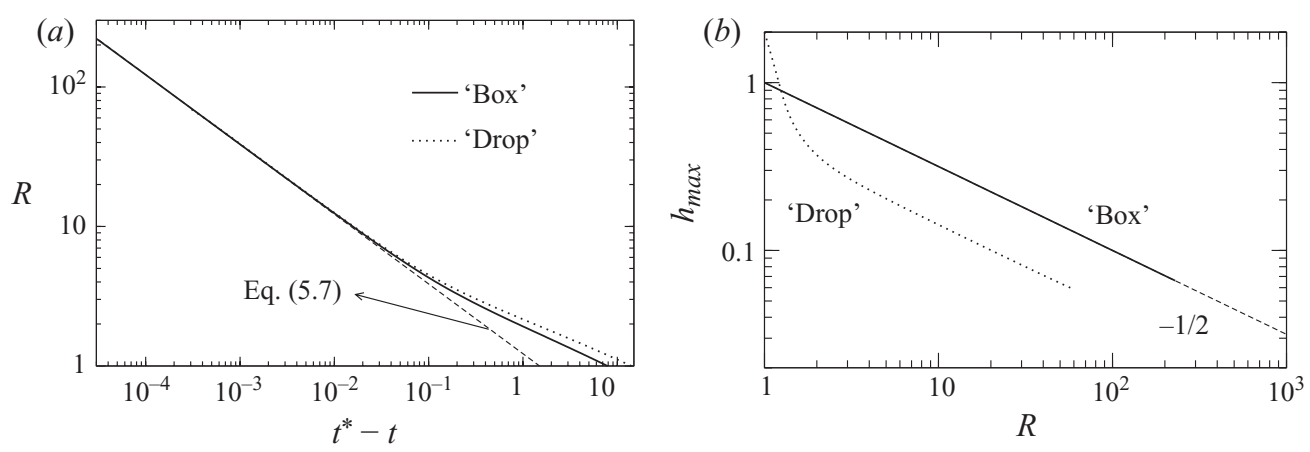

FIGURE 5. Numerical results (solid and dotted lines) for the sheet radius $R(t)$ and maximum thickness $h_{\max }(t)$ compared with the asymptotic predictions (5.7) and (5.9) (dashed lines).

whose solution is

$$
R(t)=\left(\frac{3 \mu}{2 \rho \Omega^{2}\left(t^{*}-t\right)}\right)^{1 / 2} .
$$

In figure 5(a), we compare the prediction (5.6), rewritten in dimensionless form as

$$
R(t)=\left(\frac{3}{2\left(t^{*}-t\right)}\right)^{1 / 2}
$$

with our numerical results for both the 'box' and the 'drop' initial conditions. In either case the agreement as $t \rightarrow t^{*}$ is excellent, and this allows us to estimate the critical time $t^{*}$ with high accuracy. We obtain $t^{*} \approx 7.8878$ for the 'box' and $t^{*} \approx 14.0975$ for the 'drop'.

Next, if we also neglect the contribution of the central region to the net mass of the sheet, then (5.4) determines the cross-sectional area of the rim as

$$
A=\frac{V}{2 \pi R} \propto\left(t^{*}-t\right)^{1 / 2} .
$$

It is well known (see e.g. Dewynne, Ockendon \& Wilmott 1992) that the cross-sectional aspect ratio of a Newtonian viscous thread is preserved as it stretches. It follows that both the height and the width of the rim must scale as $\left(t^{*}-t\right)^{1 / 4}$ as $t \rightarrow t^{*}$, and we deduce from (5.7) and (5.8) that the thickness of the rim should vary as $R^{-1 / 2}$.

This result can be verified from numerical simulations by plotting the scaled maximum sheet thickness $h_{\max }$ versus the scaled radius $R$, as shown in figure $5(b)$ for the two initial conditions considered in $\S 2.3$. For the 'box' initial condition, the result

$$
h_{\max }=R^{-1 / 2}
$$

is exact, as explained in the Appendix. For the 'drop' initial condition, the trend at large $R$ also matches the $-1 / 2$ power law, although now with a pre-factor that is determined numerically.

Finally, we can check a posteriori whether the simplifying assumptions that we made are justified. Our simulations indicate that the thickness in the central region follows a power-law behaviour, with $h \propto\left(t^{*}-t\right)^{\alpha}$ as $t \rightarrow t^{*}$. It is easily verified that the terms that we neglected in (5.3) and (5.4) are indeed small if and only if $\alpha>1$. As shown in $\S 4$, the choice $\alpha=1$ leads to a similarity solution that conserves mass and momentum throughout the sheet but cannot satisfy the zero-stress boundary condition. Our numerical solutions suggest $\alpha \approx 4$, in which case the contributions 


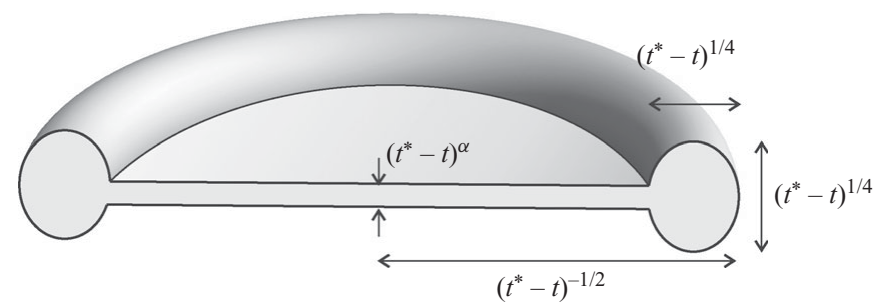

FIGURE 6. Summary of the scalings resulting from the asymptotic decomposition in the limit $t \rightarrow t^{*}$.

of the central region to (5.3) and (5.4) are certainly negligible and we can therefore expect the approximations employed in this section to be valid.

Our predicted scaling laws are summarized in figure 6 . Notice that the exponent $\alpha$ for the scaling of the thickness in the central region remains unknown thus far. We will show below how $\alpha$ may be determined by performing a more careful analysis of the asymptotic limit $t \rightarrow t^{*}$.

\section{Asymptotic analysis near the critical time}

\subsection{Analysis of the central region}

The asymptotic analysis is facilitated by introducing a small ordering parameter $\epsilon \ll 1$ characterizing the time remaining until the critical time $t^{*}$. Thus we define a new time variable $\tau$ using

$$
t=t^{*}+\epsilon \tau,
$$

while the remaining variables in the central region are scaled according to

$$
h=\epsilon^{\alpha} \tilde{h}, \quad r=\epsilon^{-1 / 2} \tilde{r}, \quad u=\epsilon^{-3 / 2} \tilde{u}, \quad \sigma=\epsilon^{-1} \tilde{\boldsymbol{\sigma}} .
$$

These scalings parameterize the symmetry that led to the similarity ansatz (4.1), and therefore exactly preserve the governing equations (2.5), (2.6) and (2.8), which are transformed to

$$
\begin{aligned}
\frac{\partial}{\partial \tau}(\tilde{r} \tilde{h})+\frac{\partial}{\partial \tilde{r}}(\tilde{r} \tilde{h} \tilde{u}) & =0, \\
\frac{\partial}{\partial \tilde{r}}\left(\tilde{r} \tilde{h} \tilde{\sigma}_{r r}\right)-\tilde{h} \tilde{\sigma}_{\theta \theta} & =-\tilde{h} \tilde{r}^{2}, \\
\tilde{\sigma}_{r r} & =4 \frac{\partial \tilde{u}}{\partial \tilde{r}}+2 \frac{\tilde{u}}{\tilde{r}}, \\
\tilde{\sigma}_{\theta \theta} & =2 \frac{\partial \tilde{u}}{\partial \tilde{r}}+4 \frac{\tilde{u}}{\tilde{r}} .
\end{aligned}
$$

\subsection{Analysis of the rim}

We examine the rim near the edge $r=R(t)$ by performing rescalings deduced from the scalings summarized in figure 6 , namely

$$
h=\epsilon^{1 / 4} \hat{h}, \quad r=R(t)+\epsilon^{1 / 4} \hat{r}, \quad R(t)=\epsilon^{-1 / 2} \hat{R}(\tau), \quad u=\dot{R}(t)+\epsilon^{-3 / 4} \hat{u}, \quad \sigma=\epsilon^{-1} \hat{\boldsymbol{\sigma}} .
$$


To leading order in $\epsilon$, the governing equations (2.5)-(2.8) reduce to

$$
\begin{aligned}
\frac{\partial}{\partial \tau}(\hat{h} \hat{R})+\frac{\partial}{\partial \hat{r}}(\hat{h} \hat{R} \hat{u}) & =0, \\
\frac{\partial}{\partial \hat{r}}\left(\hat{R} \hat{h} \hat{\sigma}_{r r}\right) & =0, \\
\hat{\sigma}_{r r} & =4 \frac{\partial \hat{u}}{\partial \hat{r}}+2 \frac{\dot{\hat{R}}}{\hat{R}}, \\
\hat{\sigma}_{\theta \theta} & =2 \frac{\partial \hat{u}}{\partial \hat{r}}+4 \frac{\hat{\hat{R}}}{\hat{R}} .
\end{aligned}
$$

From (6.9) and the boundary condition $\hat{\sigma}_{r r}=0$ at $\hat{r}=0$ we deduce that $\hat{\sigma}_{r r} \equiv 0$. It follows from (6.10) and the kinematic condition $\hat{u}=0$ at $\hat{r}=0$ that

$$
\hat{u}=-\frac{\dot{\hat{R}} \hat{r}}{2 \hat{R}},
$$

and the hoop stress is thus given by

$$
\hat{\sigma}_{\theta \theta}=\frac{3 \dot{\hat{R}}}{\hat{R}} .
$$

The mass conservation equation (6.8) takes the form

$$
\frac{\partial \hat{h}}{\partial \tau}-\left(\frac{\dot{\hat{R}} \hat{r}}{2 \hat{R}}\right) \frac{\partial \hat{h}}{\partial \hat{r}}=-\left(\frac{\dot{\hat{R}}}{2 \hat{R}}\right) \hat{h} .
$$

This hyperbolic partial differential equation (PDE) has the general solution

$$
\hat{h}=\frac{F(\chi)}{\sqrt{\hat{R}}} \quad \text { with } \quad \chi=\hat{r} \sqrt{\hat{R}},
$$

where the function $F$ is arbitrary, and may be determined only by numerical solution of an initial-value problem. We expect $\hat{h}$ to decay as $\hat{r} \rightarrow-\infty$, to match with the central region. If we suppose that $F$ varies according to

$$
F(\chi) \sim \frac{C_{1}}{(-\chi)^{m}} \text { as } \chi \rightarrow-\infty,
$$

for some constant $C_{1}$, then matching of the thickness between the central and rim regions provides the condition

$$
\epsilon^{\alpha} \tilde{h} \sim \frac{\epsilon^{(3 m+1) / 4} C_{1}}{\hat{R}^{(m+1) / 2}(\hat{R}-\tilde{r})^{m}} \quad \text { as } \quad \tilde{r} \rightarrow \hat{R} .
$$

This result tells us that the exponents $m$ and $\alpha$ must be related by

$$
m=\frac{4 \alpha-1}{3} .
$$

Since we have found that $\hat{\sigma}_{r r}$ is zero to leading order in $\epsilon$, we can seek the first perturbation by performing the rescaling $\hat{\sigma}_{r r}=\epsilon^{3 / 4} \hat{\sigma}_{r r 1}$. Then we find that $\hat{\sigma}_{r r 1}$ satisfies

$$
\frac{\partial}{\partial \hat{r}}\left(\hat{R} \hat{h} \hat{\sigma}_{r r 1}\right)=\hat{h}\left(\hat{\sigma}_{\theta \theta}-\hat{R}^{2}\right),
$$


and the solution, subject to $\hat{\sigma}_{r r 1}=0$ at $\hat{r}=0$, is

$$
\hat{\sigma}_{r r 1}=\frac{3 \dot{\hat{R}}-\hat{R}^{3}}{\hat{R}^{5 / 2} F(\chi)} \int_{0}^{\chi} F\left(\chi^{\prime}\right) \mathrm{d} \chi^{\prime} .
$$

If the area of the rim is finite, then we can set $\int_{0}^{\chi} F\left(\chi^{\prime}\right) \mathrm{d} \chi^{\prime} \rightarrow \hat{A}$ as $\chi \rightarrow-\infty$ and thus, using (6.16),

$$
\hat{\sigma}_{r r 1} \sim \frac{\left(3 \dot{\hat{R}}-\hat{R}^{3}\right) \hat{A}}{C_{1} \hat{R}^{5 / 2}}(-\hat{r} \sqrt{\hat{R}})^{m} \quad \text { as } \quad \hat{r} \rightarrow-\infty .
$$

Hence the matching condition for the stress in the central region is

$$
\epsilon^{3(m-1) / 4} \tilde{\sigma}_{r r} \sim \frac{\hat{A}\left(3 \dot{\hat{R}}-\hat{R}^{3}\right)}{C_{1} \hat{R}^{(5-m) / 2}}(\hat{R}-\tilde{r})^{m} \quad \text { as } \quad \tilde{r} \rightarrow \hat{R} .
$$

A balance in (6.22) is achieved by choosing $m=1$, which corresponds to $\alpha=1$. As we have seen in $\S 4$, this choice is unsatisfactory since it neither leads to a sensible similarity solution in the central region nor agrees with the simulations. Numerical evidence suggests that $\alpha>1$, in which case $m>1$ and the left-hand side of (6.22) is negligible to leading order in $\epsilon$. To avoid a contradiction, we must choose

$$
3 \dot{\hat{R}}=\hat{R}^{3},
$$

and hence

$$
\hat{R}(\tau)=\left(\frac{3}{-2 \tau}\right)^{1 / 2}
$$

which is equivalent to (5.6).

\subsection{Similarity solution in the central region}

The analysis carried out in $\S 5$ indicates that the mass of the sheet becomes concentrated in the rim as $t \rightarrow t^{*}$. Hence mass need not be conserved in the central region, and we can seek a more general similarity solution than in $\S 4$, with the exponent $\alpha \neq 1$. The similarity ansatz (4.1) is equivalent to

$$
\begin{aligned}
& \tilde{h}=(-\tau)^{\alpha} f(\eta), \quad \tilde{u}=(-\tau)^{-3 / 2} g(\eta), \\
& \tilde{\sigma}_{r r}=(-\tau)^{-1} S(\eta), \quad \hat{R}=(-\tau)^{-1 / 2} \ell,
\end{aligned}
$$

in our rescaled variables, with $\eta=\tilde{r} \sqrt{-\tau}$ the similarity coordinate. From (5.6) and (6.24), we have $\ell=\sqrt{3 / 2}$. Substituting $\tilde{\sigma}_{\theta \theta}=\tilde{\sigma}_{r r} / 2+3 \tilde{u} / \tilde{r}$, we reduce the governing equations (6.3)-(6.5) to the ODEs

$$
\begin{aligned}
\frac{\mathrm{d}}{\mathrm{d} \eta}\left(\eta f\left(g-\frac{\eta}{2}\right)\right) & =(\alpha-1) \eta f, \\
\eta \frac{\mathrm{d}}{\mathrm{d} \eta}(f S)+f\left(6 \frac{\mathrm{d} g}{\mathrm{~d} \eta}-S\right) & =-\eta^{2} f, \\
S & =4 \frac{\mathrm{d} g}{\mathrm{~d} \eta}+2 \frac{g}{\eta} .
\end{aligned}
$$


These equations can be rearranged into a first-order system

$$
\begin{aligned}
& \frac{\mathrm{d} g}{\mathrm{~d} \eta}=\frac{S}{4}-\frac{g}{2 \eta}, \\
& \frac{\mathrm{d} S}{\mathrm{~d} \eta}=\frac{3 g}{\eta^{2}}+\frac{4 \eta g-2 \eta^{2}+S(4 \alpha-1-S)}{2(\eta-2 g)}
\end{aligned}
$$

for $g$ and $S$, while $f$ satisfies the decoupled equation

$$
\frac{1}{f} \frac{\mathrm{d} f}{\mathrm{~d} \eta}=\frac{2 g-4 \alpha \eta+\eta S}{2 \eta(\eta-2 g)} .
$$

The free exponent $\alpha$ allows us to impose the correct boundary conditions, which was found in $\S 4$ to be impossible when $\alpha=1$. At the centre, we have zero velocity so $g(0)=0$, and the homogeneity in $f$ allows us to set $f(0)=1$ without loss of generality. At the edge $\tilde{r}=\hat{R}(\tau)$, leading-order matching conditions inferred from $\S 6.2$ imply the kinematic and zero-stress boundary conditions

$$
\tilde{u}(\hat{R}(\tau), \tau)=\dot{\hat{R}}(\tau) \quad \text { and } \quad \tilde{\sigma}_{r r}(\hat{R}(\tau), \tau)=0,
$$

which imply that

$$
g(\ell)=\frac{\ell}{2} \quad \text { and } \quad S(\ell)=0 .
$$

Reassuringly, by integrating $(6.27 c)$, we find that these lead to

$$
f(\eta) \sim \frac{\text { constant }}{(\ell-\eta)^{m}} \text { as } \eta \rightarrow \ell,
$$

where $m=(4 \alpha-1) / 3$, which is consistent with (6.18).

\subsection{Limiting behaviour and general solutions}

As $\eta \rightarrow 0$, the system (6.27) is singular and we find that there is an analytic and a non-analytic contribution, namely

$$
\begin{aligned}
& g(\eta) \sim \frac{\alpha \eta}{2}+\frac{(1-\alpha) \eta^{3}}{8(\alpha-4)}+\cdots+k \eta^{\beta}, \\
& S(\eta) \sim 3 \alpha+\frac{7(1-\alpha) \eta^{2}}{4(\alpha-4)}+\cdots+2(1+2 \beta) k \eta^{\beta-1},
\end{aligned}
$$

where $k$ is an arbitrary constant and

$$
\beta=\frac{5 \alpha-2}{2(\alpha-1)} .
$$

We recall that $\alpha$ is expected to be greater than 1 , and it follows that $\beta>5 / 2$.

We solve (6.27) numerically, starting from $\eta=0$ and using $k$ as a shooting parameter to satisfy the boundary conditions (6.29). We find that apparently legitimate solutions exist for all values of $\alpha$ greater than 1. In figure 7, we show the profiles of $f, g$ and $S$ corresponding to $\alpha=1.5,2.0,2.5,3.0,3.5$.

\subsection{Selection of $\alpha$}

One way to select $\alpha$ is to insist that the solution be analytic at $\eta=0$, so that $k$ is set to zero and $\alpha$ may then assume the role of a shooting parameter to satisfy the boundary conditions (6.29). In this case we have a similarity solution of the second kind, as classified by Barenblatt (1996), in which the exponent is selected by solving a 

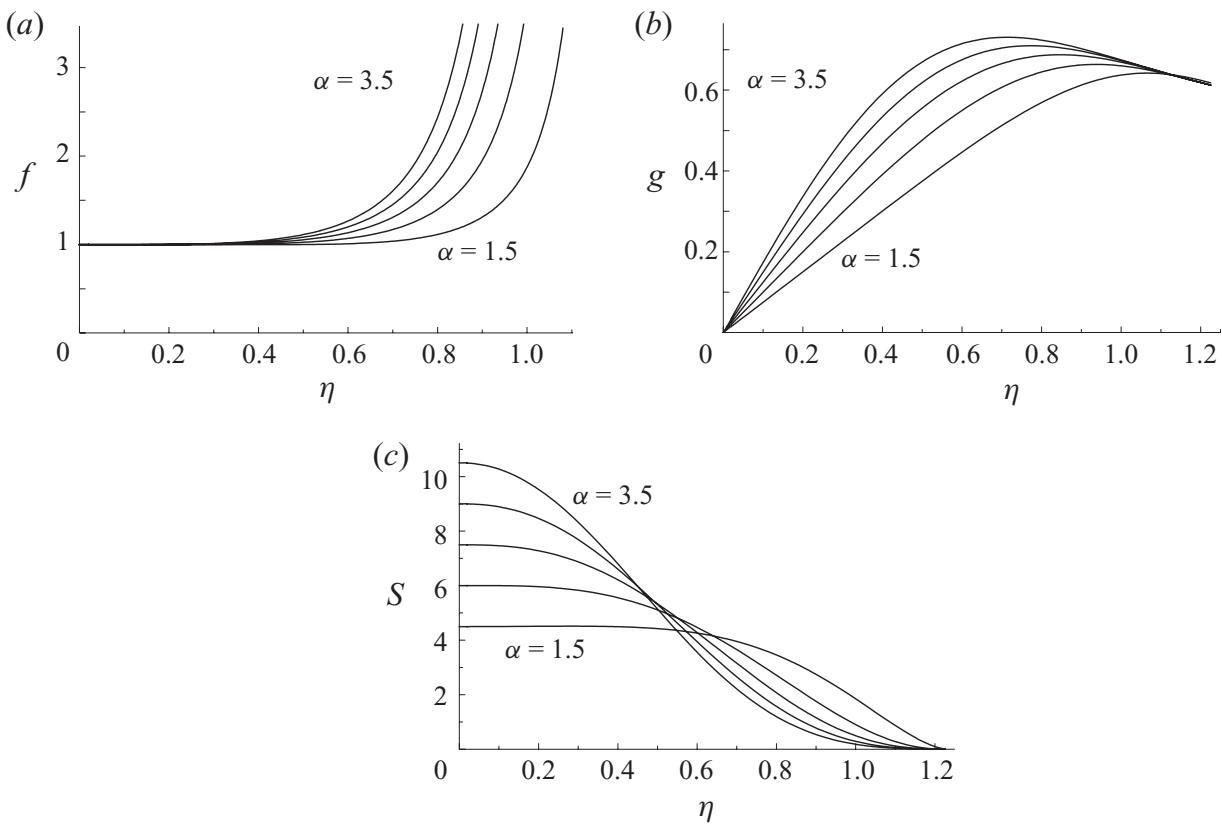

FiguRE 7. Scaled film thickness $f(\eta)$, radial velocity $g(\eta)$ and radial stress $S(\eta)$ versus similarity variable $\eta=r \sqrt{t^{*}-t}$ for values of the exponent $\alpha=1.5,2.0,2.5,3.0,3.5$.

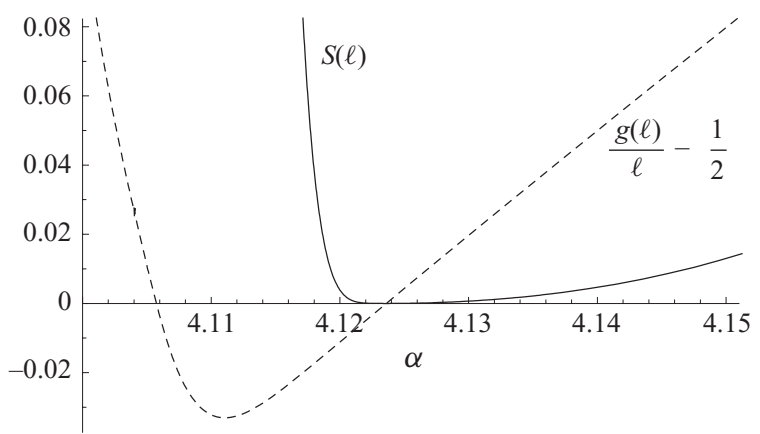

FiguRE 8. Shooting targets $g(\ell) / \ell-1 / 2$ and $S(\ell)$ versus similarity exponent $\alpha$. The curves intersect at $\alpha=4.1236$.

nonlinear eigenvalue problem, rather than being dictated by the governing equations alone. A more detailed analysis of the central region, to be reported separately, both justifies the condition $k=0$ and shows that $\alpha$ must be greater than the critical value of 4 . As shown in figure 8 , we find that both boundary conditions (6.29) are satisfied at a unique value of $\alpha \approx 4.1236$. The corresponding plot of $f(\eta)$ is shown in figure $9(a)$. We observe the anticipated structure of a thin, flat central region matching to a thick rim, with $f \rightarrow \infty$ as $\eta \rightarrow \ell=\sqrt{3 / 2}$. As shown in figure $9(b)$, the behaviour of $f(\eta)$ as $\eta \rightarrow \ell$ is consistent with (6.30), with

$$
m=\frac{4 \alpha-1}{3} \approx 5.1648 .
$$

The scaled velocity $g(\eta)$ and stress $S(\eta)$ at the selected value of $\alpha$ are plotted in figure 10 . We see that the velocity $(g)$ increases linearly from the centre of the sheet, 

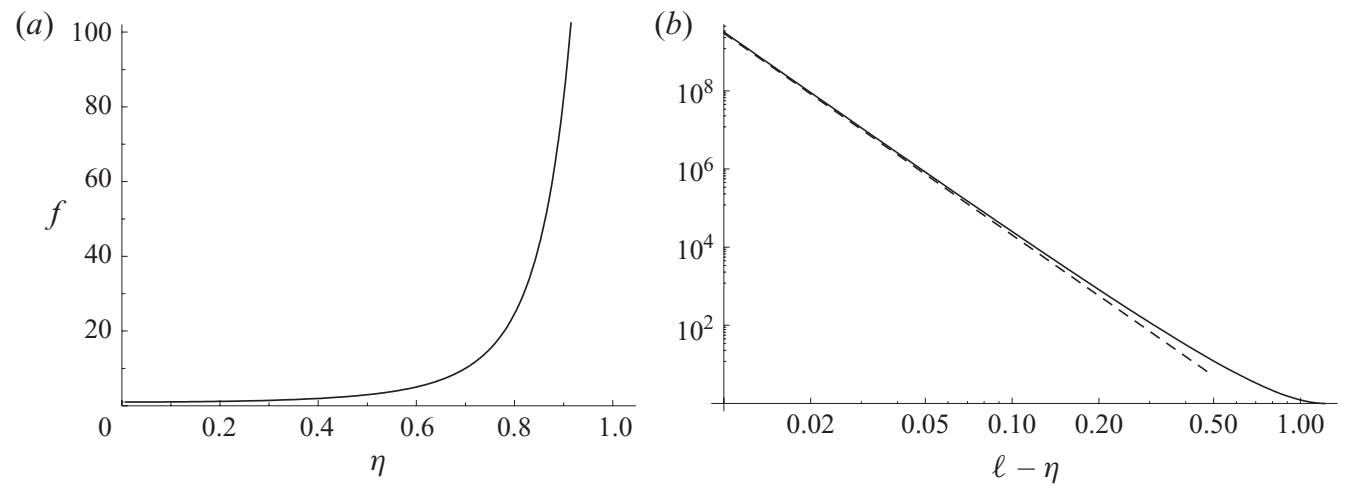

FIGURE 9. (a) Scaled film thickness $f(\eta)$ for the selected value of the exponent $\alpha \approx 4.1236$. (b) Corresponding log-log plot of $f$ versus $\ell-\eta$ (solid line) and the asymptotic behaviour $f(\eta) \sim 0.14(\ell-\eta)^{-5.1648}$ (dashed line).
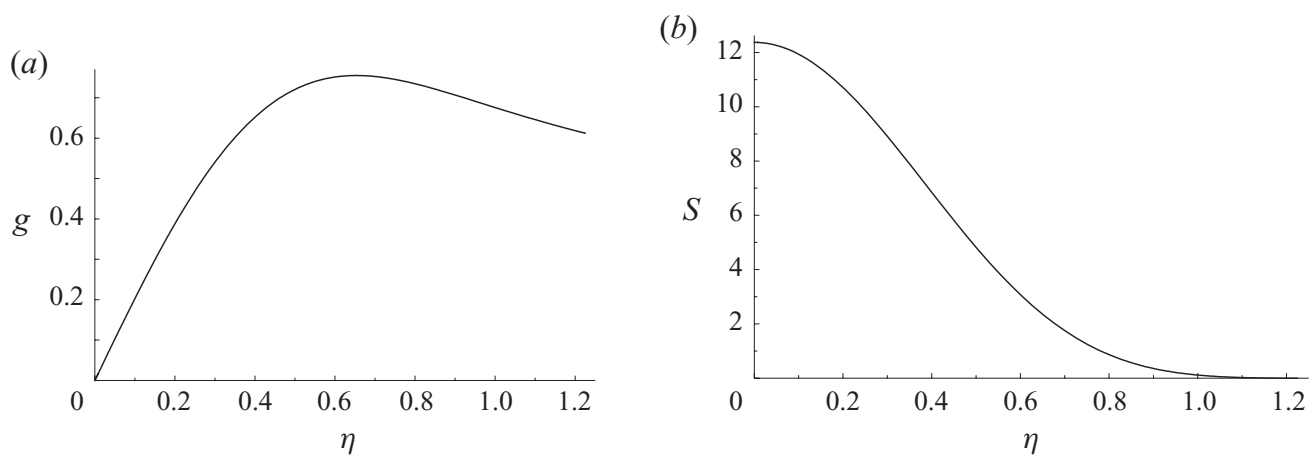

FIGURE 10. Scaled radial velocity $g(\eta)(a)$ and radial stress $S(\eta)(b)$ for the selected value of the exponent $\alpha \approx 4.1236$.

as expected. However, the condition of zero stress at the edge causes the velocity to achieve an interior maximum before decreasing towards the rim. As shown in figure 10, the scaled stress $S(\eta)$ decreases from a maximum value $3 \alpha$ at the centre of the sheet to zero at the edge.

Finally, we compare our analytical predictions to the simulations performed with the 'box' initial condition. Figure 11 shows in solid lines the minimum thickness of the sheet $h(0, t)$ versus the sheet radius $R(t)$ and the reverse time variable $t^{*}-t$, with the critical time $t^{*} \approx 7.8878$. As $t \rightarrow t^{*}$, we have superimposed in dashed lines the asymptotic behaviour using the selected value of $\alpha$, namely $h(0, t) \propto\left(t^{*}-t\right)^{4.1236}$, which is equivalent to $h(0, t) \propto R^{-8.2472}$. The visual agreement is convincing though we were only able to assess the value of $\alpha$ up to the second decimal due to numerical limitations.

\section{Incorporation of surface tension}

\subsection{Modified boundary condition}

We now show briefly how our model problem of a radially stretching spinning viscous sheet may be generalized to account for surface tension. At leading order, surface tension enters our model only through the boundary condition at the edge $r=R(t)$. 

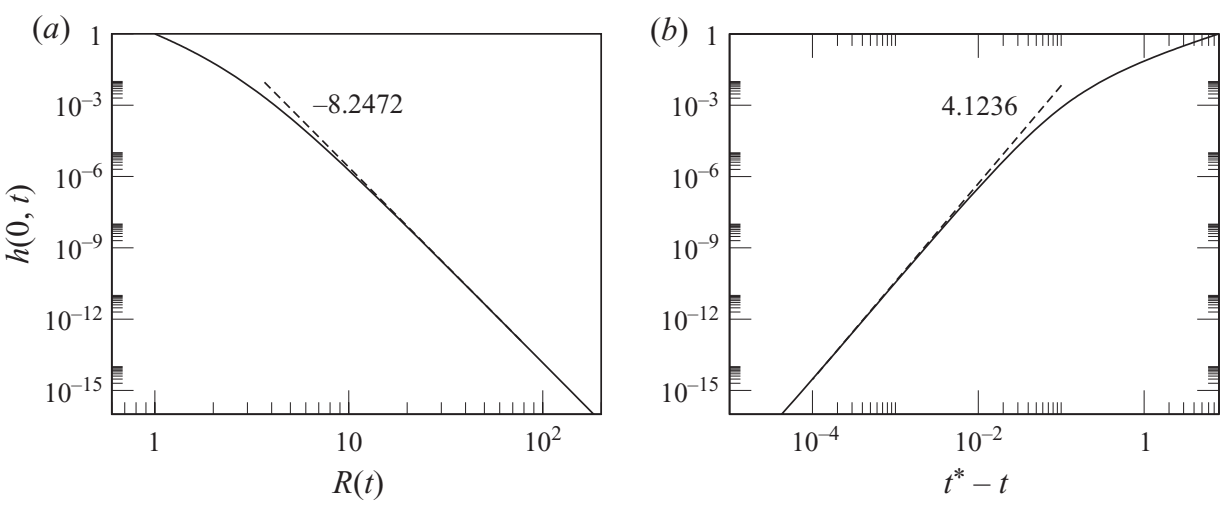

FIGURE 11. Simulations results with the 'box' initial condition showing the minimum film thickness $h(0, t)$ versus the sheet radius $R(t)$ and the reverse time variable $t^{*}-t$.

Instead of being zero, the radial stress is balanced by an inward force due to the surface tension $\gamma$ on each of the interfaces, so that

$$
h \sigma_{r r}=-2 \gamma
$$

in dimensional variables. When we non-dimensionalize according to (2.4) and use the constitutive relation (2.6), we arrive at the boundary condition

$$
2 \frac{\partial u}{\partial r}+\frac{u}{r}=-\frac{B}{h} \quad \text { at } \quad r=R(t),
$$

where $B$ is a rotational Bond number, characterizing the relative importance of capillary to centrifugal stresses and defined by

$$
B=\frac{\gamma}{\rho \Omega^{2} \bar{R}^{2} \bar{h}} .
$$

\subsection{Small-time asymptotics and simulations}

We investigate the initial effects of surface tension by performing a small-time expansion following the procedure in $\S 3$, but using (7.2) rather than the zero-stress boundary condition. We report here only the first term in the expansion, namely

$$
u(r, t) \sim \frac{r\left(7-32 B-3 r^{2}\right)}{96}, \quad h(r, t) \sim 1-\frac{\left(7-32 B-6 r^{2}\right) t}{48}, \quad R(t) \sim 1+\frac{(1-8 B) t}{24},
$$

which leads to the following results, appropriate to the limit $t \rightarrow 0$ :

$h(R(t), t) \sim 1-\frac{(1-32 B) t}{48}, \quad u(R(t), t) \sim \frac{1-8 B}{24}, \quad h(0, t) \sim 1-\frac{(7-32 B) t}{48}$.

It clearly appears that the evolution in each of these quantities experiences a reverse trend at a given value of the rotational Bond number, respectively, $B=1 / 32,1 / 8$ and $7 / 32$.

We next show in figure 12 the results of time-dependent simulations for the 'box' initial condition and four different values of the parameter $B$. As anticipated from (7.5a), for $B=0.1>1 / 32$, the maximum thickness at the rim $r=R(t)$ increases at early times, before decreasing. Figure $12(b)$ shows the corresponding behaviour when $B=0.2>1 / 8$, and we observe the sheet radius retracting initially before 
(a)

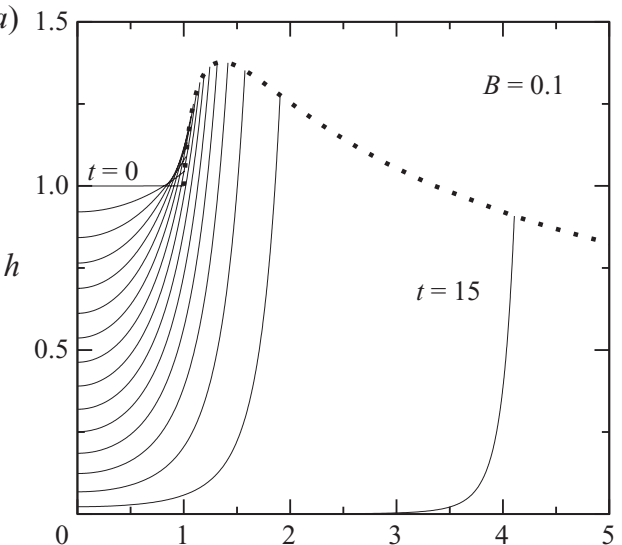

(c)

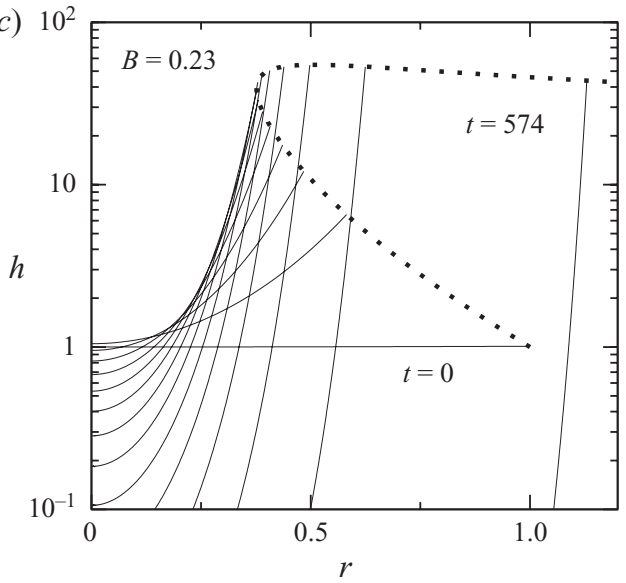

(b)

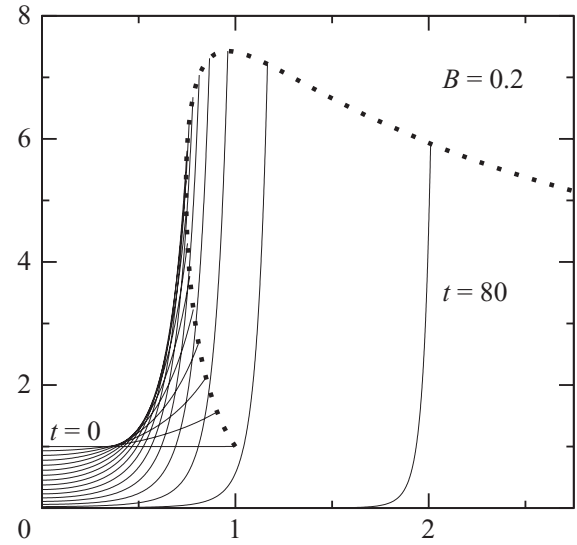

(d)

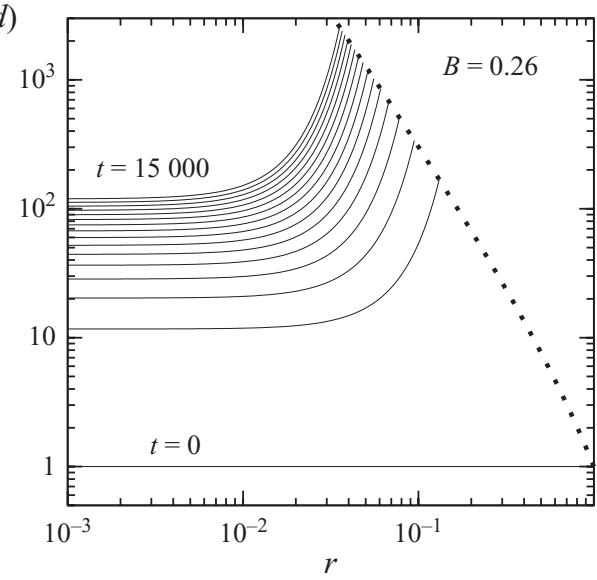

FIGURE 12. Simulation results showing thickness profiles for four different values of the rotational Bond number. The dotted line is the locus of $h_{\max }$ and the time interval is $\Delta t=1$ for $B=0.1(a), \Delta t=5$ for $B=0.2(b), \Delta t=41$ for $B=0.23(c)$ and $\Delta t=1000$ for $B=0.26(d)$.

expanding. Equation (7.5c) shows that for $B>7 / 32$ the early retraction causes the minimum thickness to increase initially, as illustrated in figure $12(c)$, where we set $B=0.23>7 / 32$. Finally, figure $12(d)$ shows that if $B$ is sufficiently large, in this case $B=0.26$, surface tension defeats the centrifugal force and the radius continues to retract indefinitely, eventually invalidating our assumption of small aspect ratio $h / R \ll 1$. In both of these final examples, the centrifugal and capillary forces are very nearly balanced, so we have to take large time intervals to observe significant variations in $h$.

These possible behaviours are summarized in figure 13, where we plot the maximum sheet thickness, achieved at the edge $r=R(t)$, versus the sheet radius $R(t)$. As $B$ is varied, the resulting curves (equivalent to the dotted curves in figure 12) sweep out a sector of phase space between $h_{\max }=R^{-1 / 2}$ when $B=0$ and $h_{\max }=R^{-2}$ when $B \rightarrow \infty$. The phase paths corresponding to $B=1 / 32,1 / 8,7 / 32,1 / 4$ divide this sector into five regions in which each of the possible behaviours identified above occurs. In region I, the behaviour is qualitatively similar to the behaviour with zero surface tension: the centrifugal force dominates, causing the sheet to stretch and thin throughout the evolution. In region II, surface tension causes an initial increase in 


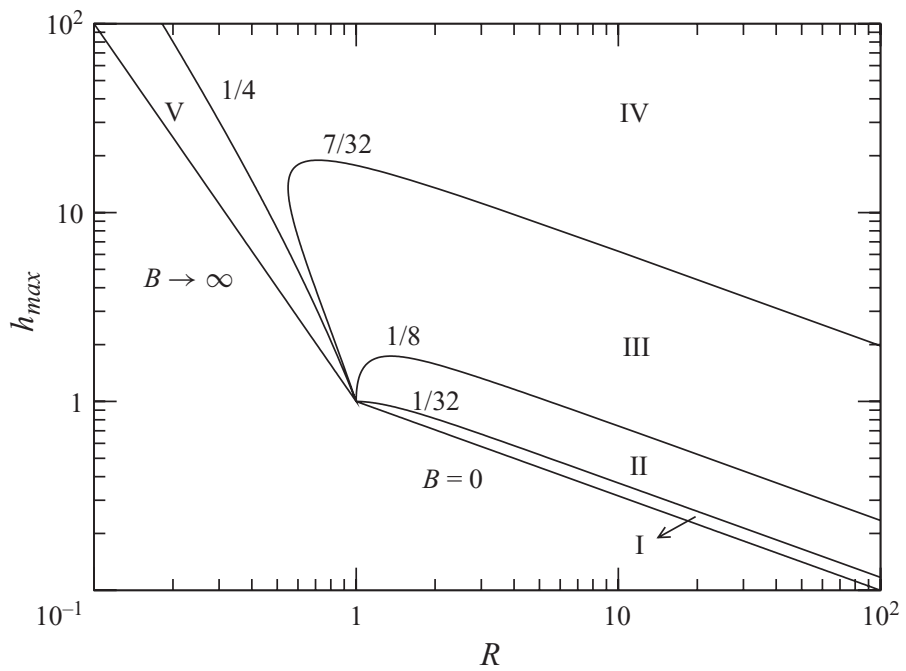

FIGURE 13. Simulation results showing the maximum thickness $h_{\max }$ versus the sheet radius $R$ for various values of the rotational Bond number $B$. These divide the phase space into five regions where distinct generic behaviours occur. I: sheet stretches and thins throughout; II: rim thickness increases initially, before decreasing; III: radius retracts initially, before expanding; IV : entire sheet thickens initially, before thinning; V: sheet retracts and thickens throughout.

$h_{\max }$ before centrifugal effects take over. A further increase in surface tension causes an initial retraction of the radius in region III and eventually an initial thickening even at the centre of the sheet in region IV. At higher values of $B$, the sheet experiences ever larger transient retraction before eventually succumbing to centrifugal stretching. Finally, when $B \geqslant 1 / 4$ surface tension dominates, and the sheet retracts indefinitely (region V).

Figure 13 applies specifically to the 'box' initial condition. However, the simulations performed in $\$ 2.3$ demonstrate that the qualitative large-time behaviour is independent of the initial data. We can thus expect the phase space corresponding to any other initial condition, for example the 'drop' from $\S 2.3$, to be similar to figure 13 , although it will not be possible in general to locate the boundaries analytically.

\subsection{Asymptotic decomposition}

As in the case of no surface tension, the simulations shown in figure 12 suggest that an asymptotic decomposition applies once the sheet has significantly expanded. Let us thus decompose the fluid into central and rim regions, as in $\S 5$. We can incorporate surface tension into the net momentum balance (5.3), which yields

$$
\frac{T}{R}+\sigma_{r r} h=\rho \Omega^{2} R A-2 \gamma
$$

Recall that $A$ denotes the cross-sectional area of the rim, and that the hoop tension $T$ is given by the constitutive relation (5.1). As in $\S 5$, we neglect the volume of fluid contained in the central region, so the net volume $V$ of the sheet is given by

$$
V=\pi \bar{R}^{2} \bar{h}=2 \pi R A .
$$




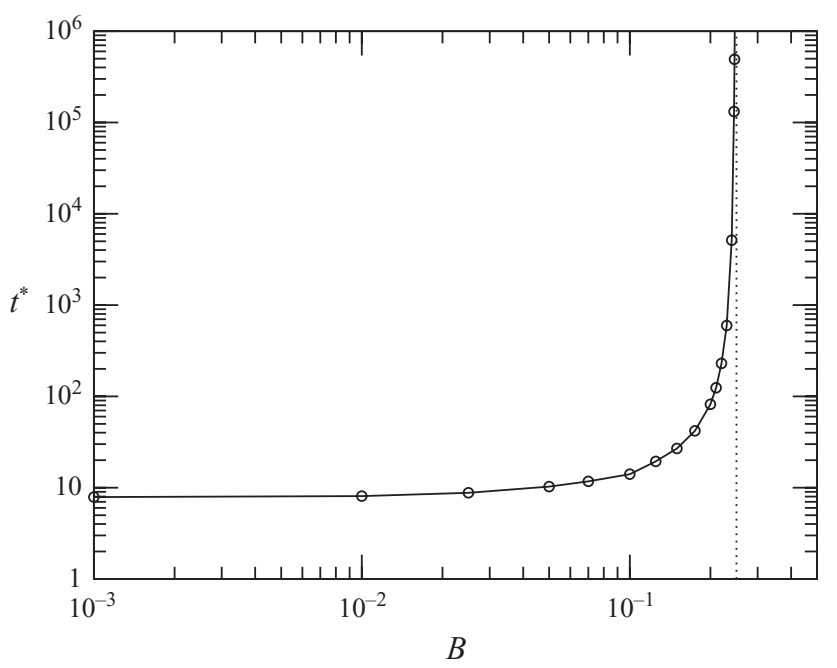

FIgURE 14. Critical time $t^{*}$ versus the rotational Bond number $B$ obtained from simulations (circles). The solid line is to guide the eye and the dashed line shows the asymptote at $\mathrm{B}=1 / 4$.

When we also neglect the tension $\sigma_{r r}$ exerted on the rim by the central region, we find that (7.6) reduces to the first-order differential equation

$$
\left(\frac{3 \mu}{\rho \Omega^{2}}\right) \frac{1}{R^{3}} \frac{\mathrm{d} R}{\mathrm{~d} t}=1-4 B
$$

The solution of $(7.8)$ is

$$
R=\left(\frac{3 \mu}{2 \rho \Omega^{2}(1-4 B)\left(t^{*}-t\right)}\right)^{1 / 2},
$$

which evidently reduces to (5.6) when $B=0$. Hence, provided $B<1 / 4$, the sheet radius still tends to infinity at some finite time $t^{*}$, which depends both on $B$ and on the initial conditions. In figure 14 we show that the critical time $t^{*}$ obtained from simulations for the 'box' initial condition is an increasing function of $B$, tending to infinity as $B \rightarrow 1 / 4$. This result agrees with the physical expectation that surface tension should oppose the stretching of the sheet.

Having found the asymptotic behaviour of the rim, we can find the corresponding solution in the central region by following the approach of $\S 6.3$. We again expect a second-kind similarity solution in which the thickness $h$ scales with $\left(t^{*}-t\right)^{\alpha}$, where $\alpha$ is to be determined numerically. We recall that this leads to the ODEs $(6.27 a)$ and $(6.27 b)$ subject to the limiting behaviour (6.31) as $\eta \rightarrow 0$. We again insist that the solution is analytic at $\eta=0$ and therefore set $k=0$, then use $\alpha$ as a shooting parameter to satisfy the boundary conditions (6.29). This procedure is identical to that used in $\S 6.3$, the only effect of surface tension being to modify $\ell$ : we infer from (7.9) the expression

$$
\ell=\sqrt{\frac{3}{2(1-4 B)}} .
$$




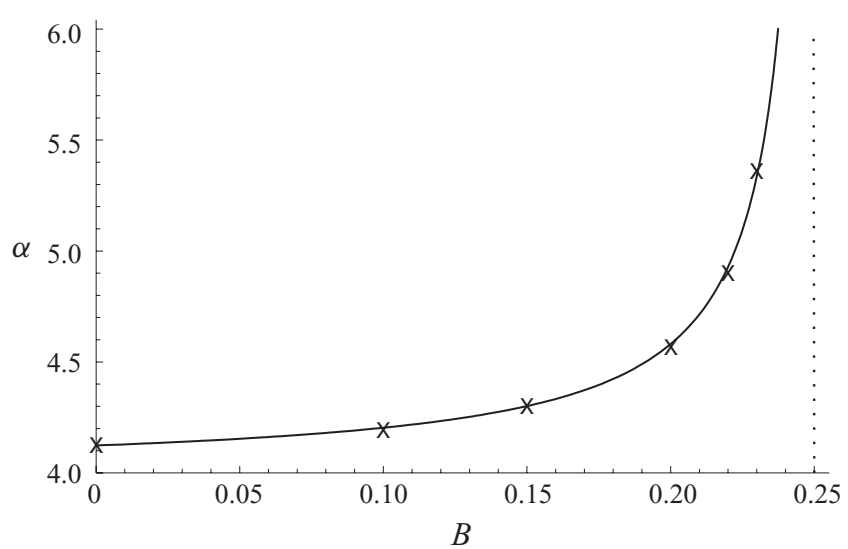

Figure 15. Similarity exponent $\alpha$ versus rotational Bond number $B$. The solid curve shows the results of the shooting procedure; the crosses are results obtained from time-dependent numerical simulations using the 'box' initial condition.

The result is plotted in figure 15 . We see that $\alpha$ is an increasing function of $B$, starting at the value 4.1236 found previously when $B=0$ and approaching infinity as $B \rightarrow 1 / 4$. The agreement with the simulations (crosses) is excellent.

\section{4. $B>1 / 4$ : similarity solution of the first kind}

If $B>1 / 4$, (7.8) suggests that surface tension overcomes the centrifugal force and causes the sheet radius to shrink over time. However, if this happens, then the hypothesized asymptotic decomposition does not arise, so the assumptions that gave rise to (7.8) are no longer valid. Instead, we find that a shrinking sheet can be described by a similarity solution of the first kind, with

$$
h=\left(t_{0}+t\right) f(\eta), \quad u=\left(t_{0}+t\right)^{-3 / 2} g(\eta), \quad R=\left(t_{0}+t\right)^{-1 / 2} \ell,
$$

where $t_{0}$ is arbitrary and the similarity coordinate is now $\eta=r \sqrt{t_{0}+t}$. Analogously to (4.3), we find that the only such solution satisfying $g(0)=0$ is

$$
f=\frac{\mathrm{e}^{\eta^{2} / 6}}{6\left(\mathrm{e}^{\mathrm{e}^{2} / 6}-1\right)}, \quad g=-\frac{\eta}{2} .
$$

In terms of our similarity variables, the stress boundary condition (7.2) reads

$$
2 \frac{\mathrm{d} g}{\mathrm{~d} \eta}+\frac{g}{\eta}=-\frac{B}{f} \quad \text { at } \quad \eta=\ell .
$$

Substituting for $f$ and $g$ from (7.12) leads to an equation for the scaled radius $\ell$, which is easily solved to give

$$
\ell=\sqrt{6 \log \left(\frac{4 B}{4 B-1}\right)} .
$$

Evidently this first-kind similarity solution successfully describes a uniformly retracting sheet provided $B>1 / 4$. Also, by evaluating $f$ at $\eta=\ell$ and using (7.14), we find that the maximum sheet thickness is given by

$$
h_{\max } \equiv h(R, t)=\left(4 B \log \left(\frac{4 B}{4 B-1}\right)\right) R^{-2},
$$


which tends to $h_{\max }=R^{-2}$ as $B \rightarrow \infty$, exactly as observed from numerical simulations in figure 13.

\section{Conclusions}

In this paper, we focused on the model problem of purely radial stretching of a circular viscous sheet by a centrifugal body force. Our model consists of coupled PDEs for the sheet thickness $h(r, t)$, radial velocity $u(r, t)$ and stress components $\sigma_{r r}(r, t)$ and $\sigma_{\theta \theta}(r, t)$. Numerical simulations show that, regardless of the initial conditions, the sheet evolves into an expanding rim attached to a very thin central film. The simulations also show that the sheet radius tends to infinity, and the thickness to zero, at some finite time $t=t^{*}$. Though we can expect the limiting behaviour of the sheet to be self-similar, it is impossible to find a similarity solution of the first kind that both conserves mass and satisfies the stress balance at the boundary.

On the other hand, we found that net mass and momentum balances in the rim enable us to explain the rim behaviour observed in numerical simulations, but do not provide information about the film thickness in the central region. To resolve this indeterminacy, we performed a formal asymptotic analysis in the limit $t \rightarrow t^{*}$. Local analysis of the rim reproduces the results of our ad hoc balance arguments and provides boundary conditions for the central region. The governing equations in the central region admit similarity solutions with $h \propto\left(t^{*}-t\right)^{\alpha}$ for any $\alpha$. By insisting that the solution be analytic at $r=0$ we obtained a unique value of $\alpha$, which is confirmed by numerical simulations. This is a similarity solution of the second kind, in which the exponent is found by solving a nonlinear eigenvalue problem. This result can occur because the governing PDE for $h(r, t)$ is hyperbolic, and hence remembers features of the initial conditions. In particular, it can be shown that the solution must remain analytic at $r=0$ if it is so initially, and this justifies our criterion for selecting the similarity exponent.

Future development of this problem includes analysis of the stability of the stretching sheet to azimuthal perturbations. Indeed, the analogous problem of spin coating is known to be susceptible to a fingering instability, and the radially expanding sheet formed by the impact of a jet on a flat substrate is known to break up. One can also include non-Newtonian effects, which may help to stabilize the system and are obviously important if materials such as bread doughs (pizza) are to be modelled.

In this paper, we neglected the contribution of acceleration to the fluid inertia, while retaining the centrifugal body force. This approximation is valid provided the rotational Reynolds number $\rho \Omega R^{2} / \mu$ is small. Hence, as the radius $R$ increases, the acceleration terms must eventually become significant, and one can show that they act to cut off the finite-time blow-up predicted by our model, leading instead to rapid exponential growth.

Our selected value of the exponent $\alpha \approx 4.1236$ corresponds to $h(0, t) \sim R^{-8.2472}$, which explains why the central region becomes extremely thin for moderately large values of $R$. To obtain accurate estimates of $\alpha$, we pushed our simulations (see e.g. figure 11) down to unphysically small values of $h$. In reality, other physical effects not included in our model, for example, inertia or non-Newtonian rheology, would probably become important long before the sheet becomes so thin. Eventually, if the thickness decreases to $100 \mathrm{~nm}$ or so, intermolecular effects may come into play and cause the sheet to rupture in the central region. In this case, a process of outward sheet retraction should take place, as recently studied by Savva \& Bush (2009). 
The second-kind similarity solution found in the present problem seems to occur because of a degeneracy which precludes a similarity solution that both conserves mass and satisfies the stress boundary condition. Among other problems where similarity solutions of the second kind are encountered for similar reasons, we mention: the spreading groundwater mound of liquid in a porous medium (Wagner 2005); the spreading of insoluble surfactant at the free surface of a deep fluid layer (Jensen 1995); dynamics and rupture of planar electrified liquid sheets (Tilley, Petropoulos \& Papageorgiou 2001); the flow in a cylindrical container with a rotating end wall (Muite 2004); the problem of rotary honing (a two-dimensional variant of the Taylor paint-scraper problem) where a similarity solution of the second kind is contained within a similarity solution of the first kind (Hills \& Moffatt 2000).

Examples of rupture events previously studied in the literature, like the pinching of a thread (Papageorgiou 1995; Renardy 2001) or the line rupture of a sheet (e.g. Vaynblat et al. 2001a) are qualitatively quite different from the behaviour we observed in the present paper. Both van der Waals sheet rupture and capillary thread rupture are 'local', with the thickness tending to zero generically at a single point. In contrast, centrifugal breakup is 'non-local', in that the entire central region thins at the same rate until it breaks up everywhere simultaneously, at the same critical time $t^{*}$.

We thank Saint-Gobain Recherche for support of this investigation and Rémi Magnard for technical support with Comsol. We also thank John Bush for suggesting the inclusion of surface tension and forwarding a preprint of Savva \& Bush (2009). P.D.H. gratefully acknowledges the hospitality of the Harvard School of Engineering and Applied Sciences, where he was on sabbatical during this project. B.S. thanks the Brussels Region for the funding through the programme 'Brains Back to Brussels'.

\section{Appendix. Transformation to Lagrangian variables}

To facilitate both asymptotic analysis and numerical simulations, it is helpful to transform the problem to Lagrangian variables that move with the stretching sheet. We seek solutions for $h$ and the Eulerian variable $r$ as functions of $t$ and a Lagrangian spatial variable $\xi$ defined by

$$
\frac{\partial}{\partial t}(r(\xi, t))=u(r(\xi, t), t), \quad r(\xi, 0)=\xi .
$$

One advantage of this approach is that the moving domain $r \in[0, R(t)]$ is mapped to a fixed domain $\xi \in[0,1]$.

We discover from the mass-conservation equation (2.8) that

$$
\frac{\partial}{\partial t}\left(r h \frac{\partial r}{\partial \xi}\right)=0
$$

and from the initial conditions we deduce that

$$
\frac{\partial r}{\partial \xi}=\frac{\xi h_{0}(\xi)}{r h} .
$$

Thus $r(\xi, t)$ is determined instantaneously, given the initial condition $h_{0}(\xi)$ and the boundary condition

$$
r(0, t)=0 .
$$


In terms of the Lagrangian variables, the momentum equation (2.5) may be written in the form

$$
\frac{\partial}{\partial t}\left(\sqrt{r} \frac{\partial h}{\partial \xi}\right)=\frac{\xi h_{0}(\xi)}{4} \sqrt{r}
$$

This inhomogeneous wave equation for $h(\xi, t)$ requires one initial condition, namely

$$
h(\xi, 0)=h_{0}(\xi),
$$

and one boundary condition. To obtain this, we note that the radial stress may be written as

$$
\sigma_{r r}=-2 \frac{\partial}{\partial t}\left(\log \left(r h^{2}\right)\right)
$$

Hence the imposition of zero stress at the edge leads to the boundary condition

$$
h(1, t)=\frac{h_{0}(1)}{\sqrt{r(1, t)}},
$$

which explains the exact agreement of (5.9) with the 'box' simulations.

The coupled partial differential equations (A 3) and (A 5), along with the boundary conditions (A 4), (A 6) and (A 8), determine $h$ and $r$ as functions of $\xi$ and $t$, and therefore give us a parametric description of the sheet thickness at each instant. The velocity and stress are then given by (A 1) and (A 7), while the radius of the sheet may be inferred a posteriori from

$$
R(t)=r(1, t) .
$$

\section{REFERENCES}

Barenblatt, G. I. 1996 Scaling, Self-similarity, and Intermediate Asymptotics. Cambridge University Press.

Buckmaster, J. D., NACHMAn, A. \& Ting, L. 1975 The buckling and stretching of a viscida. J. Fluid Mech. 69, 1-20.

Cao, F., Khayat, R. E. \& Puskas, J. E. 2005 Effect of inertia and gravity on the draw resonance in high-speed film casting of Newtonian fluids. Intl J. Solids Struct. 42, 5734-5757.

Dewynne, J. N., Ockendon, J. R. \& Wilmott, P. 1992 A systematic derivation of the leading-order equations for extensional flows in slender geometries. J. Fluid Mech. 244, 323-338.

EgGers, J. 1997 Nonlinear dynamics and breakup of free-surface flows. Rev. Mod. Phys. 69, 865-930.

Eggers, J. \& Villermaux, E. 2008 Physics of liquid jets. Rep. Prog. Phys. 71, 036601.

England, P. \& McKenzie, D. 1982 A thin viscous sheet model for continental deformation. Geophys. J. R. Astron. Soc. 70, 295-321.

Erneux, T. \& Davis, S. H. 1993 Nonlinear rupture of free films. Phys. Fluids A 5, 1117-1122.

Hills, C. P. \& Moffatt, H. K. 2000 Rotary honing: a variant of the Taylor paint-scraper problem. J. Fluid Mech. 418, 119-135.

Howell, P. D. 1996 Models for thin viscous sheets. Eur. J. Appl. Math. 7, 321-343.

Huang, J. C. P. 1970 The break-up of axisymmetric liquid sheets. J. Fluid Mech. 43, 305-319.

IDA, M. P. \& Miksis, M. J. 1996 Thin film rupture. Appl. Math. Lett. 9, 35-40.

JENSEN, O. E. 1995 The spreading of insoluble surfactant at the free surface of a deep fluid layer. J. Fluid Mech. 293, 349-378.

Muite, B. K. 2004 The flow in a cylindrical container with a rotating end wall at small but finite Reynolds number. Phys. Fluids 16, 3614-3626.

Ng, T. S. K., McKinley, G. H. \& Padmanabhan, M. 2006 Linear to non-linear rheology of wheat flour dough. Tech. Rep. 06-P-08. Massachusetts Institute of Technology.

Papageorgiou, D. T. 1995 On the breakup of viscous liquid threads. Phys. Fluids 7, 1529-1544.

Pearson, J. R. A. 1985 Mechanics of Polymer Processing. Elsevier. 
Pilkington, L. A. B. 1969 Review lecture. The float glass process. Proc. R. Soc. Lond. A 314, 1-25. Renardy, M. 2001 Finite time breakup of viscous filaments. Z. Angew. Math. Phys. 52, 881.

Ribe, N. M. 2002 A general theory for the dynamics of thin viscous sheets. J. Fluid Mech. 457, 255-283.

Savart, F. 1833 Mémoire sur le choc d'une veine liquide lancée contre un plan circulaire. Ann. Chim. 54, 56-87.

Savva, N. \& Bush, J. W. M. 2009 Viscous sheet retraction. J. Fluid Mech. 626, 211-240.

Scheid, B., Quiligotti, S., Tran, B. \& Stone, H. 2009 Lateral shaping and stability of a stretching viscous sheet. Eur. Phys. J. B 68, 487-494.

TAYlor, G. I. $1959 a$ The dynamics of thin sheets of fluid. Part II. Waves on fluid sheets. Proc. R. Soc. Lond. A 253, 296-312.

TAYLOR, G. I. $1959 b$ The dynamics of thin sheets of fluid. Part III. Disintegration of fluid sheets. Proc. R. Soc. Lond. A 253, 313-321.

Tilley, B. S., Petropoulos, P. G. \& Papageorgiou, D. T. 2001 Dynamics and rupture of planar electrified liquid sheets. Phys. Fluids 13, 3547-3563.

VAYNBlat, D., Lister, J. R. \& Witelski, T. P. 2001 a Rupture of thin viscous films by van der Waals forces: evolution and self-similarity. Phys. Fluids 13, 1130-1140.

Vaynblat, D., Lister, J. R. \& Witelski, T. P. $2001 b$ Symmetry and self-similarity in rupture and pinchoff: a geometric bifurcation. Eur. J. Appl. Math. 12, 209-232.

WAGNER, B. 2005 An asymptotic approach to second-kind similarity solutions of the modified porous-medium equation. J. Engng Math. 53, 201-220. 